\title{
Noise-driven neuromorphic tuned amplifier
}

\author{
Duccio Fanelli, ${ }^{1,2}$ Francesco Ginelli, ${ }^{3}$ Roberto Livi, ${ }^{1,2}$ Niccoló Zagli, ${ }^{1}$ and Clement Zankoc ${ }^{1,2}$ \\ ${ }^{1}$ Dipartimento di Fisica e Astronomia and CSDC, Università degli Studi di Firenze, via G. Sansone 1, 50019 Sesto Fiorentino, Italy \\ ${ }^{2}$ INFN Sezione di Firenze, via G. Sansone 1, 50019 Sesto Fiorentino, Italy \\ ${ }^{3}$ SUPA, Institute for Complex Systems and Mathematical Biology, Kings College, University of Aberdeen, Aberdeen AB24 $3 U E$, \\ United Kingdom
}

(Received 19 August 2017; published 26 December 2017)

\begin{abstract}
We study a simple stochastic model of neuronal excitatory and inhibitory interactions. The model is defined on a directed lattice and internodes couplings are modulated by a nonlinear function that mimics the process of synaptic activation. We prove that such a system behaves as a fully tunable amplifier: the endogenous component of noise, stemming from finite size effects, seeds a coherent (exponential) amplification across the chain generating giant oscillations with tunable frequencies, a process that the brain could exploit to enhance, and eventually encode, different signals. On a wider perspective, the characterized amplification process could provide a reliable pacemaking mechanism for biological systems. The device extracts energy from the finite size bath and operates as an out of equilibrium thermal machine, under stationary conditions.
\end{abstract}

DOI: 10.1103/PhysRevE.96.062313

\section{INTRODUCTION}

Living systems implement and execute an extraordinary plethora of computational tasks [1,2]. The brain, with its structural and functional architecture, represents an emblematic example of hierarchic self-organization [3]: elementary units, the neurons, act much like instruments of an orchestra, which combine diverse timbres to create harmonious symphonies. Neurons team up to process external stimuli from a number of sources and integrate the information to yield, from neurons to mind, different cognitive faculties. Identifying the coarse grained modules that exert, from bottom to up, pivotal neuronal functions constitutes a goal of paramount importance. On the other hand, the brain and its unraveled secrets could inspire novel biomimetics technologies to adaptively handle complex problems. In this paper, we investigate the intertwined stochastic dynamics of two populations of excitatory and inhibitory units, arranged in a directed lattice [4-7]. The endogenous noise [8-10] seeds a coherent amplification across the chain generating giant oscillations with tunable frequencies, a process that the brain could exploit to enhance, and eventually encode, different signals. The same scheme could be in principle invoked so as to provide a reliable stochastic pacemaking for a large gallery of biological systems. On the other hand, it could inspire the design of novel manmade detectors capable of reacting to spatially distributed low intensity alerts.

Simple deterministic models can be devised so to exemplify, at the mesoscopic level, the prototypical evolution of excitatory and inhibitory units, organized in two mutually competing populations [11]. This is for instance the case of the celebrated Wilson-Cowan (WC) model of excitatory and inhibitory neurons [12-14]. By replicating the WC model on each patch of a supposedly heterogeneous network, and assuming that local activation gets modulated by adjacent populations, yields a spatially extended framework where coherent patterns of activation can organize and flow. Stochastic perturbation can however play a remarkable contribution [15-22]. Finite size effects manifest in particular as an endogenous source of disturbance, which ultimately reflects the inherent discrete- ness of the scrutinized medium $[4,8,9,23]$. It is indeed the endogenous component of noise which fuels the process of tuned amplification that we shall hereafter illustrate, working with a minimal model for discrete agents in mutual interaction, via excitatory and inhibitory loops, as recently introduced and discussed in [24]. ${ }^{1}$ The model is formulated in terms of a birth-death stochastic process. In the idealized deterministic limit, it converges to a set of rate equations for the densities of active excitatory and inhibitory neurons, reminiscent of the WC type.

Apart for neuroscience related applications, the mechanism of stochastic pacemaker that we will here characterize considerably extends beyond the classical concept of quasicycles, as originally envisaged by Bartlett [23] and subsequently studied by McKane and Newman [9]. Quasicycles are regular oscillations in the concentration of the interacting species, as seeded by the demographic fluctuations, and disappear when the number of individuals is strictly infinite. The oscillations are however small in amplitude. This latter scales in fact as the inverse of the (square root of the) system size (or volume) of the scrutinized system, an inherent constraint that has been so far deemed as unavoidable. Also the power spectrum of the obtained signal is generally broad and the stochastic driven oscillations consequently irregular. By working in a spatially extended framework and building on the asymmetry of the imposed coupling, we fill these gaps: giant oscillations (with tunable frequencies) are produced when moving along the chain that defines the spatial skeleton of the model. While growing in size, the oscillations get progressively more clean an observation that we shall analytically quantify.

\footnotetext{
${ }^{1}$ In [24] a symmetric network of couplings is assumed: this working hypothesis hinders the coherent amplification mechanism, that we shall here thoroughly explore. Assuming a directed lattice as the backbone for the spatial interaction is just a choice that enables for the analytical calculation to be carried out. The identified phenomenon is however more general and applies to a wide range of settings, as mentioned in the conclusions.
} 


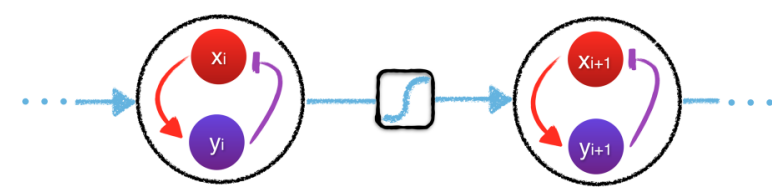

FIG. 1. Schematic layout of the neuromorphic circuit.

\section{STOCHASTIC MODEL}

Let us start by introducing the model, as presented in [24]. Label $X_{i}\left(Y_{i}\right)$, with $i=1,2, \ldots, \Omega$, the excitatory (inhibitory) agents belonging to the mean-field interacting patch (hereafter also called node) $i$ of volume $V_{i}$. The patches are organized in a one-dimensional lattice, as depicted in Fig. 1, with directional couplings. Individual elements are subject to the following birth and death chemical reactions:

$$
\begin{array}{llllll}
\emptyset & \stackrel{f\left[s_{x_{i}}\right]}{\longrightarrow} & X_{i} & \emptyset & \stackrel{f\left[s_{y_{1}}\right]}{\longrightarrow} & Y_{i} \\
X_{i} & \stackrel{1}{\longrightarrow} \emptyset & & Y_{i} & \stackrel{1}{\longrightarrow} & \emptyset,
\end{array}
$$

where $\emptyset$ denotes an infinite reservoir; $f(s)=1 /[1+\exp (-s)]$ is a sigmoid function which captures the saturating response of neurons to external stimuli $[4,12,13]$. Further

$$
\begin{aligned}
& s_{x_{i}}=-r\left(y_{i}-\frac{1}{2}\right)+D\left(x_{i-1}-x_{i}\right)-D\left(y_{i-1}-y_{i}\right), \\
& s_{y_{i}}=r\left(x_{i}-\frac{1}{2}\right)+D\left(x_{i-1}-x_{i}\right)-D\left(y_{i-1}-y_{i}\right),
\end{aligned}
$$

where $x_{i}=n_{X_{i}} / V_{i}\left(y_{i}=n_{Y_{i}} / V_{i}\right)$ is the concentration of active excitators (inhibitors) on node $i ; n_{X_{i}}$ and $n_{Y_{i}}$ respectively identify the number of elements of type $X$ and $Y$ on patch $i$. The intensity of the couplings between adjacent populations is set by the positive defined parameter $D$. The directed nearest neighbors interaction is mediated by the nonlinear filter $f$, a scheme assumed in the literature to plausibly represent synaptic interactions. $r>0$ acts as a control parameter of the local dynamics. In the uncoupled limit $(D=0)$, a large population of inhibitors damps the corresponding population of excitators, on the same patch. Similarly, a large excitatory population stimulates a local growth of the inhibitors.

Introduce $P(\boldsymbol{v}, t)$ to label the probability for the system to be in state $\boldsymbol{v}=\left(x_{1}, y_{1}, x_{2}, y_{2}, \ldots, x_{\Omega}, y_{\Omega}\right)$ at time $t$. Transitions from one state to another are dictated by the above chemical equations. $T\left(\boldsymbol{v}^{\prime} \mid \boldsymbol{v}\right)$ stands for the transition rate from state $\boldsymbol{v}$ to state $\boldsymbol{v}^{\prime}$, compatible with the former. The dynamics of the system is governed by a master equation $[8,25]$ which can be cast in the generic form $\frac{\partial}{\partial t} P(\boldsymbol{v}, t)=\sum_{v^{\prime}} T\left(\boldsymbol{v} \mid \boldsymbol{v}^{\prime}\right) P\left(\boldsymbol{v}^{\prime}, t\right)-$ $T\left(\boldsymbol{v}^{\prime} \mid \boldsymbol{v}\right) P(\boldsymbol{v}, t)$.

One can then seek to approximate the exact master equation via the Kramers Moyal expansion [25-29] assuming large enough $V_{1}$ and $\gamma_{i}=V_{i} / V_{1}=O(1)$. To this end we begin by rewriting the master equation as

$$
\frac{\partial P}{\partial t}=\left(\Gamma_{1}+\Gamma_{2}\right) P
$$

where the operators $\Gamma_{1}$ and $\Gamma_{2}$ are given by

$$
\begin{aligned}
& \Gamma_{1}=\sum_{i=1}^{\Omega}\left(\epsilon_{x_{i}}^{+}-1\right) T\left(x_{i}-\frac{1}{V_{i}} \mid \boldsymbol{v}\right)+\left(\epsilon_{x_{i}}^{-}-1\right) T\left(x_{i}+\frac{1}{V_{i}} \mid \boldsymbol{v}\right), \\
& \Gamma_{2}=\sum_{i=1}^{\Omega}\left(\epsilon_{y_{i}}^{+}-1\right) T\left(y_{i}-\frac{1}{V_{i}} \mid \boldsymbol{v}\right)+\left(\epsilon_{y_{i}}^{-}-1\right) T\left(y_{i}+\frac{1}{V_{i}} \mid \boldsymbol{v}\right)
\end{aligned}
$$

and the step operators are defined as

$$
\begin{aligned}
& \epsilon_{x_{i}}^{ \pm} f\left(x_{i}, y_{i}\right)=f\left(x_{i} \pm \frac{1}{V_{i}}, y_{i}\right), \\
& \epsilon_{y_{i}}^{ \pm} f\left(x_{i}, y_{i}\right)=f\left(x_{i}, y_{i} \pm \frac{1}{V_{i}}\right) .
\end{aligned}
$$

For large enough $V_{i}$, one can approximate the step operators as

$$
\begin{aligned}
& \epsilon_{x_{i}}^{ \pm} \approx 1 \pm \frac{1}{V_{i}} \frac{\partial}{\partial x_{i}}+\frac{1}{2 V_{i}^{2}} \frac{\partial^{2}}{\partial x_{i}^{2}}, \\
& \epsilon_{y_{i}}^{ \pm} \approx 1 \pm \frac{1}{V_{i}} \frac{\partial}{\partial y_{i}}+\frac{1}{2 V_{i}^{2}} \frac{\partial^{2}}{\partial y_{i}^{2}} .
\end{aligned}
$$

By inserting the above expressions into the master equation, performing the calculations and introducing $\tau=\frac{t}{V_{1}}$ yields the following Fokker-Planck equation:

$$
\frac{\partial P}{\partial \tau}=-\sum_{i=1}^{2 \Omega} \frac{\partial}{\partial v_{i}} A_{i} P+\sum_{i, j=1}^{2 \Omega} \frac{1}{2 V_{1}} \frac{\partial^{2}}{\partial v_{i} \partial v_{j}} B_{i j} P,
$$

where

$$
\boldsymbol{A}=\left(\begin{array}{c}
\cdots \\
\cdots \\
\frac{1}{\gamma_{i}}\left[T\left(x_{i}+\frac{1}{V_{i}} \mid \boldsymbol{v}\right)-T\left(x_{i}-\frac{1}{V_{i}} \mid \boldsymbol{v}\right)\right] \\
\frac{1}{\gamma_{i}}\left[T\left(y_{i}+\frac{1}{V_{i}} \mid \boldsymbol{v}\right)-T\left(y_{i}-\frac{1}{V_{i}} \mid \boldsymbol{v}\right)\right] \\
\cdots \\
\cdots
\end{array}\right)
$$

and $\boldsymbol{B}$ is a diagonal $2 \Omega \times 2 \Omega$ matrix made of $\Omega$ distinct $2 \times 2$ blocks $\boldsymbol{B}_{i}$ with entries

$$
\begin{aligned}
& \left(\boldsymbol{B}_{i}\right)_{11}=\frac{1}{\gamma_{i}^{2}}\left[T\left(x_{i}+\frac{1}{V_{i}} \mid \boldsymbol{v}\right)+T\left(x_{i}-\frac{1}{V_{i}} \mid \boldsymbol{v}\right)\right], \\
& \left(\boldsymbol{B}_{i}\right)_{12}=\left(\boldsymbol{B}_{i}\right)_{21}=0, \\
& \left(\boldsymbol{B}_{i}\right)_{22}=\frac{1}{\gamma_{i}^{2}}\left[T\left(y_{i}+\frac{1}{V_{i}} \mid \boldsymbol{v}\right)+T\left(y_{i}-\frac{1}{V_{i}} \mid \boldsymbol{v}\right)\right],
\end{aligned}
$$

for $\mathrm{i}=1, \ldots, \Omega$.

By making use of the explicit form of the transition rates,

$$
\begin{aligned}
& T\left(x_{i}+\frac{1}{V_{i}} \mid \boldsymbol{v}\right)=f\left(s_{x_{i}}\right), \\
& T\left(x_{i}-\frac{1}{V_{i}} \mid \boldsymbol{v}\right)=x_{i}, \\
& T\left(y_{i}+\frac{1}{V_{i}} \mid \boldsymbol{v}\right)=f\left(s_{y_{i}}\right), \\
& T\left(y_{i}-\frac{1}{V_{i}} \mid \boldsymbol{v}\right)=y_{i},
\end{aligned}
$$


one readily obtains, from the above Fokker-Planck equation, the equivalent Langevin equations:

$$
\begin{aligned}
& \frac{d}{d \tau} x_{i}=\frac{1}{\gamma_{i}}\left[f\left(s_{x_{i}}\right)-x_{i}\right]+\frac{1}{\gamma_{i} \sqrt{V_{1}}} \sqrt{x_{i}+f\left(s_{x_{i}}\right)} \lambda_{i}^{(1)}, \\
& \frac{d}{d \tau} y_{i}=\frac{1}{\gamma_{i}}\left[f\left(s_{y_{i}}\right)-y_{i}\right]+\frac{1}{\gamma_{i} \sqrt{V_{1}}} \sqrt{y_{i}+f\left(s_{y_{i}}\right)} \lambda_{i}^{(2)},
\end{aligned}
$$

where $\lambda=\left(\lambda_{1}^{(1)}, \lambda_{1}^{(2)}, \lambda_{2}^{(1)}, \lambda_{2}^{(2)}, \ldots, \lambda_{\Omega}^{(1)}, \lambda_{\Omega}^{(2)}\right)$ is a Gaussian stochastic variable with zero mean and correlator $\left\langle\lambda_{i}^{(l)} \lambda_{j}^{(m)}\right\rangle=$ $\delta_{i j} \delta_{l m} \delta\left(\tau-\tau^{\prime}\right)$. Here, $\delta_{i j}\left(\delta_{l m}\right)$ is the Kronecker symbol. In the thermodynamic limit $V_{1} \rightarrow \infty$, the above stochastic equations reduce to a deterministic system which admits the homogenous fixed point $x^{*}=y^{*}=1 / 2, \forall i$. The stability of the fixed point can be assessed by computing the eigenvalues of the $2 \Omega \times 2 \Omega$ Jacobian matrix $\boldsymbol{J}$, that we shall explicitly report in the Appendixes. We will begin by assuming nodes of identical capacity $V_{i}=V$, which entails $\gamma_{i}=1$. The spectrum of $\boldsymbol{J}$ is hence degenerate, owing to the peculiar structure of the block matrix. Two eigenvalues read $\lambda_{1,2}=-1 \pm i r / 4$ and coincide with those obtained when working with just one patch $(\Omega=1)$. The other eigenvalues are $\lambda_{3,4}=\left[-1 \pm \sqrt{\left(\frac{r}{8}\left(D-\frac{r}{2}\right)\right)}\right]$, each with multiplicity $\Omega-1$. As expected, $\lambda_{3,4}$ converge to $\lambda_{1,2}$, when $D \rightarrow 0$. Based on this relation, we can immediately conclude that the homogeneous fixed point is linearly stable provided $D<D_{c} \equiv \frac{r}{2}+\frac{8}{r}$. Importantly, the eigenvalues are complex for $D<r / 2<D_{c}$, an observation that plays a crucial role for what it follows. Summing up, for $D<r / 2$, the deterministic system displays, at equilibrium, a uniform level of activity, across the lattice, for both excitators and inhibitors.

We now specialize on the stochastic, finite size dynamics, and hence assume $V_{1}$ to be finite. When $D=0$ the stochastic trajectories on each node are formally disentangled. Excitators (inhibitors) execute almost regular oscillations about their deterministic equilibrium. These oscillations are termed in the literature quasicycles $[9,10]$ and follow a resonant amplification process triggered by the endogenous component of noise [23]. The amplitude of the oscillations scales as $1 / \sqrt{V_{1}}$ and the associated frequency approximately reads $\omega_{0}=r / 4$, the imaginary part of the Jacobian eigenvalues in the uncoupled, $D=0$, setting. A remarkably different scenario is faced when turning the coupling active. We will in particular operate for $D<r / 2$, the homogeneous fixed point being therefore stable. The degenerate component of the Jacobian spectrum returns an additional frequency $\omega_{1}=\sqrt{\frac{r}{8}\left(\frac{r}{2}-D\right)}$ which can be continuously modulated, in the range $\left[0, \omega_{0}\right]$, as function of $D$. This observation is central to understanding the emerging stochastic dynamics: the internal noise seeds in fact giant quasicycles, with tunable frequency and growing amplitude across the lattice. The system spontaneously behaves as an effective, stochastic driven pacemaker, a nontrivial selforganized dynamics that we shall hereafter demonstrate.

\section{AMPLIFICATION MECHANISM}

Under the linear noise approximation (LNA) [8,25], stochastic effects act as linear deviations from the deterministic solution. We hence set

$$
\begin{aligned}
& x_{i}=x^{*}+\frac{\xi_{i}}{\sqrt{V_{i}}}=x^{*}+\frac{1}{\sqrt{V_{1}}} \frac{\xi_{i}}{\sqrt{\gamma_{i}}}, \\
& y_{i}=y^{*}+\frac{\eta_{i}}{\sqrt{V_{i}}}=y^{*}+\frac{1}{\sqrt{V_{1}}} \frac{\eta_{i}}{\sqrt{\gamma_{i}}},
\end{aligned}
$$

where $\left(\xi_{i}, \eta_{i}\right)$ stand for the stochastic perturbation. Inserting the above ansatz in the nonlinear function $f(\cdot)$ and performing the expansion at the first order in $1 / \sqrt{V_{1}}$ yields

$$
\begin{aligned}
& f\left(s_{x_{i}}\right) \approx \frac{1}{2}+\frac{1}{4 \sqrt{V_{1}}}\left[-\frac{r \eta_{i}}{\sqrt{\gamma_{i}}}+D \Delta \xi_{i}-D \Delta \eta_{i}\right]+O\left(\frac{1}{V_{1}}\right), \\
& f\left(s_{x_{i}}\right) \approx \frac{1}{2}+\frac{1}{4 \sqrt{V_{1}}}\left[\frac{r \xi_{i}}{\sqrt{\gamma_{i}}}+D \Delta \xi_{i}-D \Delta \eta_{i}\right]+O\left(\frac{1}{V_{1}}\right),
\end{aligned}
$$

where $\Delta \xi_{i} \equiv\left(\frac{\xi_{i-1}}{\sqrt{\gamma_{i-1}}}-\frac{\xi_{i}}{\sqrt{\gamma_{i}}}\right)$ and $\Delta \eta_{i} \equiv\left(\frac{\eta_{i-1}}{\sqrt{\gamma_{i-1}}}-\frac{\eta_{i}}{\sqrt{\gamma_{i}}}\right)$. Here, use has been made of the condition $f(0)=1 / 2$ and $f^{\prime}(0)=$ $f(0)[1-f(0)]=1 / 4, f^{\prime}(\cdot)$ labeling the derivative of $f(\cdot)$. The nonlinear amplitudes that characterize the multiplicative noise in the Langevin equations, see Eqs. (2), reduce to constant factors under the linear noise approximation. Specifically,

$$
\begin{aligned}
& \frac{1}{\gamma_{i} \sqrt{V_{1}}} \sqrt{x_{i}+f\left(s_{x_{i}}\right)} \approx \frac{1}{\gamma_{i} \sqrt{V_{1}}}+O\left(\frac{1}{V_{1}}\right), \\
& \frac{1}{\gamma_{i} \sqrt{V_{1}}} \sqrt{y_{i}+f\left(s_{y_{i}}\right)} \approx \frac{1}{\gamma_{i} \sqrt{V_{1}}}+O\left(\frac{1}{V_{1}}\right) .
\end{aligned}
$$

Building on the above one can write the linear version of the nonlinear Langevin equations. To this end, we introduce $\zeta=\left(\xi_{1}, \eta_{1}, \ldots, \xi_{\Omega}, \eta_{\Omega}\right)$ and write

$$
\frac{d}{d \tau} \zeta_{i}=\sum_{j=1}^{2 \Omega} J_{i j} \zeta_{j}+\rho_{i}
$$

where $J_{i j}$ are the entries of the Jacobian matrix $\boldsymbol{J}$ and $\rho_{i}$ is Gaussian noise with zero mean and correlator $\left\langle\rho_{i}(\tau) \rho_{j}\left(\tau^{\prime}\right)\right\rangle=$ $\mathcal{B}_{i j} \delta\left(\tau-\tau^{\prime}\right) . \mathcal{B}_{i j}$ are the entries of the diffusion matrix $\mathcal{B}$ defined as

$$
\mathcal{B}=\left(\begin{array}{ccccc}
\boldsymbol{I}_{2 \times 2} & 0 & 0 & 0 & 0 \\
0 & \frac{1}{\gamma_{2}} \boldsymbol{I}_{2 \times 2} & 0 & 0 & 0 \\
0 & 0 & \frac{1}{\gamma_{3}} \boldsymbol{I}_{2 \times 2} & 0 & 0 \\
0 & 0 & \ddots & \ddots & 0 \\
0 & 0 & 0 & 0 & \frac{1}{\gamma_{\Omega}} \boldsymbol{I}_{2 \times 2}
\end{array}\right),
$$

where $\boldsymbol{I}_{2 \times 2}$ stands for the $2 \times 2$ identity matrix. The linear Langevin equations (5) are equivalent to the following FokkerPlanck equation for the probability distribution function $\Pi(\zeta, \tau)$ of fluctuations:

$$
\frac{\partial}{\partial \tau} \Pi=-\sum_{i=1}^{2 \Omega} \frac{\partial}{\partial \zeta_{i}}\left[(J \zeta)_{i} \Pi\right]+\frac{1}{2} \sum_{i, j=1}^{2 \Omega} \frac{\partial^{2}}{\partial \zeta_{i} \partial \zeta_{j}} \mathcal{B}_{i j} \Pi .
$$

It is worth emphasizing that the above equation could be also derived by performing a van Kampen expansion of the original master equation for the probability $P(\boldsymbol{v}, t)$. 
(a)

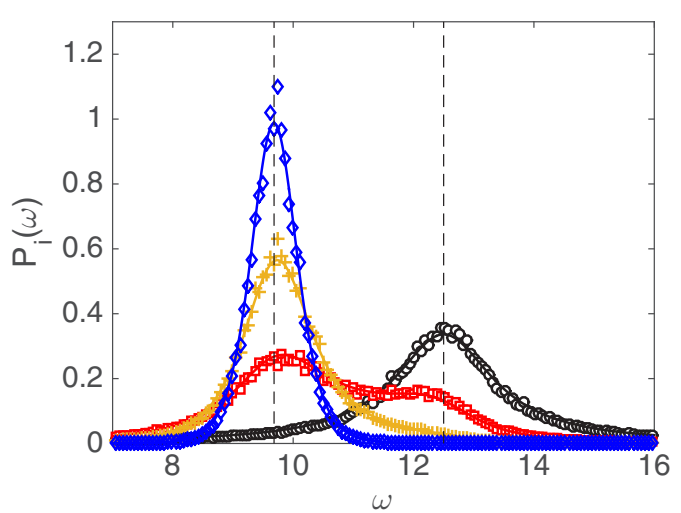

(b)

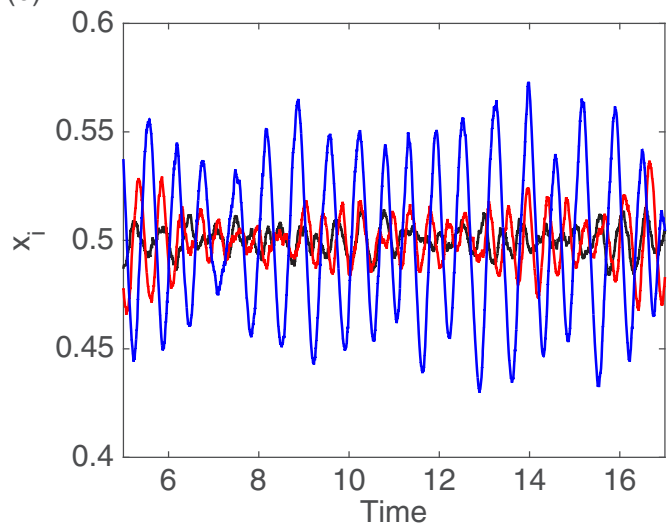

FIG. 2. Panel (a): the theoretical power spectrum $P_{i}(\omega)$ (7) for excitators $(X)$, for $i=1$ (black), 2 (red), 3 (orange), and 5 (blue) is plotted with a solid line. Symbols refer to the power spectra computed from averaging independent realizations of the stochastic dynamics (2). In the Appendixes we show that the nonlinear Langevin dynamics (2) returns results which are in excellent agreement with exact stochastic simulations based on Gillespie algorithm [32]. The rightmost vertical dashed line is traced at $\omega_{0}$ and the leftmost at $\omega_{1}$. Here $r=50, D=10$, and $V=10^{6}$. Panel (b): stochastic trajectories on different nodes. Noisy self-sustained oscillations of modest amplitude are displayed on the first node of the lattice (black line). The amplitude of the oscillations grows steadily across the chain (red line on node 2 and blue line on node 8 ) and the oscillations become progressively more regular.

To proceed in the analysis, denote $\tilde{\zeta}_{i}(\omega)$ the Fourier transform of $\zeta_{i}(t)$. Then, one gets $\tilde{\zeta}_{i}(\omega)=\sum_{j=1}^{2 \Omega} \Phi_{i j}^{-1}(\omega) \tilde{\rho}_{j}(\omega)$, where $\Phi_{i j}=-J_{i j}-i \omega \delta_{i j}$. One can hence calculate the power spectrum of fluctuations on node $i$ :

$$
P_{i}(\omega)=\left\langle\tilde{\zeta}_{i}(\omega) \tilde{\zeta}_{i}^{*}(\omega)\right\rangle=\sum_{l=1}^{2 \Omega} \Phi_{i l}^{-1}(\omega)\left(\Phi^{\dagger}\right)_{l i}^{-1}(\omega) .
$$

In the above equation, for the sake of simplicity, we have assumed $V_{i}=V_{1}$, hence $\gamma_{i}=1$, which implies replacing $\mathcal{B}$ with the identity matrix. In the first panel of Fig. 2 the (normalized) power spectrum of excitator fluctuations on different nodes is plotted. Symbols refer to the numerical integration of Eqs. (2) [30], while the solid lines follow the theoretical estimate (7). The power spectrum on the first node (circles, black online) is centered in $\omega_{0}$ (rightmost vertical dashed line). The power spectrum on the second node (squares, red online) displays a bimodal profile. A second peak emerges in correspondence of $\omega_{1}$ (leftmost vertical dashed line). Moving along the chain (pluses and diamonds), the bump in $\omega_{0}$ fades away, while the peak in $\omega_{1}$ gains in potency and gets progressively more localized. Individual trajectories as obtained on different nodes are superposed in the panel (b) of Fig. 2: the amplification can be clearly appreciated by eye inspection. A movie is also annexed as Supplemental Material [31] to better visualize the displayed amplification process. Under the linear noise approximation, the maximum of the power spectrum diverges exponentially (not shown) along the chain. At the same time the width of the bell in $\omega_{1}$ becomes narrower and the profile converges asymptotically to a deltalike distribution. Beatings and other spurious modulations are therefore progressively filtered, as moving along the chain and building on the idealized linear approach: the system is hence predicted to eventually behave as a veritable pacemaker. However, nonlinear terms do matter and eventually balance the growth, as predicted within the linear scenario. Indeed, the process of amplification is expected to come to a halt when the oscillations get large enough so as to feel the boundary at $x_{i} \simeq 0\left(y_{i} \simeq 0\right)$.

In simple physical terms, each species can be assimilated (rigorously, in the linear regime) to a damped harmonic oscillator. This latter is forced out of equilibrium, owing to the interaction with the populations that belong to the preceding node. The forcing, as instigated by nearest neighbor (directed) couplings and backed by finite size noise, gets progressively more intense as the perturbation propagates along the chain, node after node. This observation sits at the root of the detected amplification process.

To shed further light on to this mechanism and eventually quantify the amplification grade under the linear noise approximation, we set to consider the distribution of fluctuations $\Pi(\zeta, t)$ around the deterministic equilibrium. As it is shown in the Appendixes, $\Pi(\zeta, t)$ obeys a Fokker-Planck equation which can be self-consistently derived via the van Kampen system size expansion. The solution of the Fokker-Planck equation is a multivariate Gaussian that we can univocally characterize in terms of the associated first and second moments. It is immediate to show that the first moment converges to zero. We focus instead on the $2 \Omega \times 2 \Omega$ family of second moments, defined as $\left\langle\zeta_{l} \zeta_{m}\right\rangle=\int \zeta_{l} \zeta_{m} \Pi d \zeta$. A straightforward calculation (see the Appendixes) yields

$$
\begin{aligned}
\frac{d}{d \tau}\left\langle\zeta_{l}^{2}\right\rangle & =+2\left\langle\zeta_{l}(J \zeta)_{l}\right\rangle+\mathcal{B}_{l l}, \\
\frac{d}{d \tau}\left\langle\zeta_{l} \zeta_{m}\right\rangle & =\left\langle\zeta_{l}(J \zeta)_{m}\right\rangle+\left\langle\zeta_{m}(J \zeta)_{l}\right\rangle
\end{aligned}
$$

for respectively the diagonal and off-diagonal $(l \neq m)$ moments. The stationary values of the moments can be analytically computed by setting to zero the time derivatives on the left hand side of Eqs. (8) and solving the linear system that is consequently obtained. We are in particular interested in accessing 
(a)

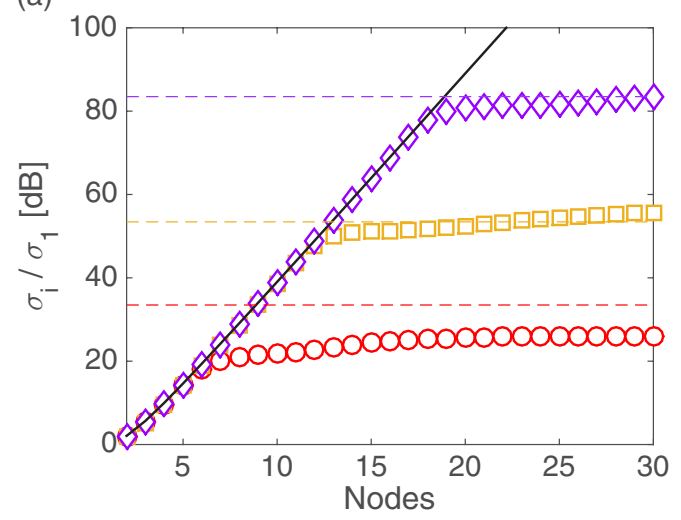

(b)

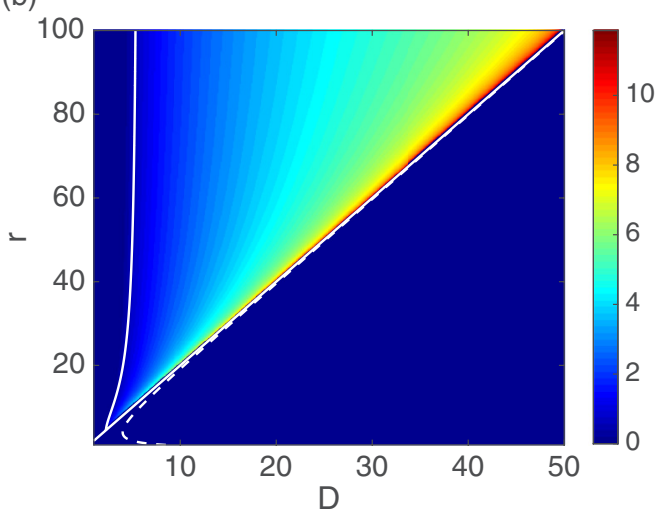

FIG. 3. Panel (a): $\sigma_{i} / \sigma_{1}$ [in decibel (dB) logarithmic scale] is plotted against the index which identified the ordering of the nodes along the lattice. Data refer to the excitatory species. The solid line stands for the analytical estimate obtained under linear noise approximation. The amplification process is clearly exponential. Symbols refer to direct integration of equations (2), for different choices of the volume $V_{1}\left(10^{6}\right.$, circles; $10^{12}$, squares; $10^{18}$, diamonds). The horizontal dashed lines show where the linear estimate predicts $\sigma_{i} \simeq 1 / 2$, namely when saturation is theoretically expected to occur. Here $D=10$ and $r=50$. Panel (b): the rate of exponential amplifications (for the excitators) is depicted in the plane $(r, D)$. The domain where the amplification is expected to take place is delimited by the two solid curves. The dashed line refers to $D_{c}$ vs $r$.

$\sigma_{i}=\sqrt{\left\langle\zeta_{i}^{2}\right\rangle}$, the standard deviation of the fluctuations as displayed, around the deterministic equilibrium, on node $i$. The value of $\sigma_{i}$, normalized to $\sigma_{1}$ and expressed in decibel (dB), is plotted against the nodes index along the lattice in Fig. 3, panel (a). The data refer to the excitatory species. The solid line stands for the analytical estimate that implements the above strategy. Remarkably, the standard deviation of the fluctuations grows exponentially along the chain. Symbols refer instead to direct integration of Eqs. (2), for different choices of the volume $V_{1}$. The agreement with the theory prediction based on the linear ansatz is excellent over a finite portion of the chain. When $\sigma_{i} \simeq 1 / 2$ (horizontal dashed line) the system senses the boundary, nonlinearities come into play and induce the observed saturation. By increasing $V_{1}$, one reduces the amplitude of the endogenous fluctuations: the signal has therefore to travel through a larger set of contiguous nodes before the amplitude of the oscillation can hit the extinction edge. As a consequence, the linear approximation holds over a larger portion of the scrutinized chain. The rate of exponential growth (relative to the excitators species), as predicted by the linear theory, is plotted with an appropriate color code, in the reference parameters plane $(r, D)$; see panel (b) of Fig. 3. The amplification takes place within a bounded region in $(r, D)$, as delimited by the two solid (white) lines. The straight line that sets the rightmost frontier of the amplification domain is obtained as $r=D / 2$, namely the condition of existence of a complex imaginary part in the degenerate eigenvalues $\lambda_{3,4}$ (which in turn select the frequency $\omega_{1}$ to be amplified). The boundary that delimits the region of interest on the left follows a closed analytical estimate, obtained by truncating long range correlations in the estimate of the multivariate moments to nearest neighbors (details in the Appendixes). The dashed (white) line refers to $D_{c}$ versus $r$ and it is depicted for the sake of completeness. Similar results (not shown) apply to the inhibitors.

\section{OTHER CONFIGURATIONS AND POSSIBLE APPLICATIONS}

The noise assisted amplification process that we have here characterized is very flexible and can be configured in different schemes. By augmenting the volumes of the nodes along the chain, and so consequently tuning the additional control parameters $\gamma_{i}$, we can amplify virtually any harmonic of $\omega_{0}$ (or, alternatively, $\omega_{1}$ ). This possibility is demonstrated in the Appendixes. The amplification pattern can also take place on a frequency comb, by appropriately assigning the relative weight $\gamma_{i}$; see the Appendixes. Following a similar strategy, it is also possible to focus the amplification on frequencies larger than $\omega_{0}$.

In all inspected cases, the self-sustained amplification is fueled by the inherent component of noise, stemming from finite size corrections. At variance, one could imagine assembling a device that operates in the deterministic $V_{i} \rightarrow \infty$ limit. If $D<D_{c}$, the system is frozen in its homogeneous equilibrium, the concentration of both $x_{i}$ and $y_{i}$ being identical to $1 / 2$ on each node. Assume now that a perturbation, limited in time and modest in amplitude, hit on the first node. For demonstrative purposes we exemplify the perturbation as a noisy signal, drawn from a random uniform distribution. The disturbance propagates along the chain and gets magnified, as follows the scheme that we outlined above, exciting on site oscillations at a given frequency $\omega_{1}$, that could be freely tuned by acting, e.g., on $D$. Such an apparatus could efficaciously act as a signal detector, a possibility that is demonstrated in the movie annexed as Supplemental Material [31]. Even more interesting, one could foresee the possibility of assembling a detector that exploits parallel lines of detection. On each line a different value of the coupling $D$ could be enforced. In doing so, from the trace of the amplified signal at the end of the chain (processed with a standard frequency analyzer), it could be possible to identify the node (hence the chain) where 
the perturbation hit. This observation opens up the perspective to define a class of detectors that could spatially resolve low intensity alerts.

\section{ON A THERMODYNAMICAL INTERPRETATION}

As a final point, we will elaborate on a consistent thermodynamic interpretation of the process that underlies the spontaneous generation of giant quasioscillations. Our analysis follows the approach pioneered by [33-36] to study the thermodynamics of far-from-equilibrium systems, which are microscopically amenable to stochastic continuous time Markovian processes. Given the probability density $P(\boldsymbol{v}, \tau)$ that satisfies the Fokker-Planck equation (1), we define the entropy $S(\tau)$ as

$$
S(\tau)=-\int P(\boldsymbol{v}, \tau) \ln P(\boldsymbol{v}, \tau) d \boldsymbol{v} .
$$

By deriving with respect to time $\tau$ the previous equation, one gets

$$
\frac{d S}{d \tau}=-\int \frac{\partial P}{\partial \tau}(\ln P+1) d v=\int \sum_{i} \frac{\partial I_{i}}{\partial v_{i}}(\ln P+1) d v
$$

and, integrating by parts,

$$
\frac{d S}{d \tau}=-\sum_{i} \int I_{i} \frac{\partial}{\partial v_{i}} \ln P d \boldsymbol{v}
$$

where the current $I_{i}=A_{i} P-\frac{1}{2} B_{i i} \frac{\partial}{\partial v_{i}} P$ is the probability density current associated to the Fokker-Planck equation (1). On the other hand,

$$
\frac{\partial}{\partial v_{i}} \ln P=\frac{2}{B_{i i}} A_{i}-\frac{2}{B_{i i}} \frac{I_{i}}{P}
$$

and finally

$$
\frac{d S}{d \tau}=\Pi_{S}-\Phi_{S}
$$

where

$$
\begin{aligned}
\Pi_{S} & =\sum_{i} \frac{2}{B_{i i}} \int \frac{I_{i}^{2}(\boldsymbol{v}, \tau)}{P(\boldsymbol{v}, \tau)}, \\
\Phi_{S} & =\sum_{i} \frac{2}{B_{i i}} \int A_{i}(\boldsymbol{v}) I_{i}(\boldsymbol{v}, \tau) d \boldsymbol{v} .
\end{aligned}
$$

$\Pi_{S}$ is positive definite and can be interpreted as the production rate of entropy due to the nonconservative forces $A_{i}$. $\Phi_{S}$ can take in principle any sign. When $\Phi_{S}>0$, the entropy flows from the system to the environment. At equilibrium $I_{i}=0$, which implies $\Pi_{S}=\Phi_{S}=0$. A nontrivial stationary solution exists which corresponds to setting $\Pi_{S}=\Phi_{S} \neq 0$. This is equivalent to imposing $\sum \frac{\partial}{\partial v_{i}} I_{i}=0$. The condition of solenoidal current, $\boldsymbol{\nabla} \cdot \boldsymbol{I}=0$, is indeed met when the FokkerPlanck equation attains its nontrivial dynamical equilibrium $\left(I_{i} \neq 0\right)$. In other words, the observed amplification stems from a genuine noise-driven out-of-equilibrium process, the neuromorphic device working under stationary operating conditions.
By making use of the definition of the current, and performing an integration by parts, one gets

$$
\begin{aligned}
\Phi_{S} & =\sum_{i} \frac{2}{B_{i i}} \int A_{i} I_{i} d \boldsymbol{v}=\sum_{i} \frac{2}{B_{i i}} \int\left(A_{i}^{2} P-\frac{B_{i i}}{2} A_{i} \frac{\partial}{\partial v_{i}} P\right) \\
& =\sum_{i} \frac{2}{B_{i i}} \int\left(A_{i}^{2} P+\frac{B_{i i}}{2} P \frac{\partial}{\partial v_{i}} A_{i}\right) \\
& =\sum_{i}\left(\frac{2}{B_{i i}}\left\langle A_{i}^{2}\right\rangle+\left\langle\frac{\partial}{\partial v_{i}} A_{i}\right\rangle\right) .
\end{aligned}
$$

The above formula con be employed to determine the (nonlinear) entropy production rate $\Pi_{S}\left(=\Phi_{S}\right)$, displayed by the system in stationary conditions. To gain analytical insight we can proceed with a direct estimate of $\Pi_{S}$ (and hence $\Phi_{S}$ ) that builds on the linear noise approximation. In this case we can write

$$
\Phi_{S}=\sum_{i}\left(\frac{2}{\mathcal{B}_{i i}}\left\langle f_{i}^{2}\right\rangle+\left\langle\frac{\partial}{\partial \zeta_{i}} f_{i}\right\rangle\right),
$$

where the nonconservative force is now $f_{i}=(J \zeta)_{i}$. Recalling that

$$
\sum_{i}\left\langle\frac{\partial}{\partial \zeta_{i}} f_{i}\right\rangle=\sum_{i}\left\langle\sum_{j} J_{i j} \frac{\partial \zeta_{j}}{\partial \zeta_{i}}\right\rangle=\sum_{i}\left\langle J_{i i}\right\rangle=\operatorname{Tr}(J),
$$

we can write

$$
\Phi_{S}=\sum_{i, j, k} \frac{2}{\mathcal{B}_{i i}} J_{i j} J_{i k}\left\langle\zeta_{j} \zeta_{k}\right\rangle+\operatorname{Tr}(J)
$$

Define then the correlation matrix $C_{i j}=\left\langle\zeta_{i} \zeta_{j}\right\rangle$ and write

$$
\begin{aligned}
\Phi_{S} & =2 \sum_{i, j, k} \frac{1}{\mathcal{B}_{i i}} J_{i j} J_{i k} C_{j k}+\operatorname{Tr}(J) \\
& =2 \sum_{i} \frac{1}{\mathcal{B}_{i i}}\left(J C J^{t}\right)_{i i}+\operatorname{Tr}(J) .
\end{aligned}
$$

In Fig. 4 the entropy production rate $\Pi_{S}\left[=\Phi_{S}\right.$, as given by formula (11)] is plotted (solid line) versus the lattice node, an

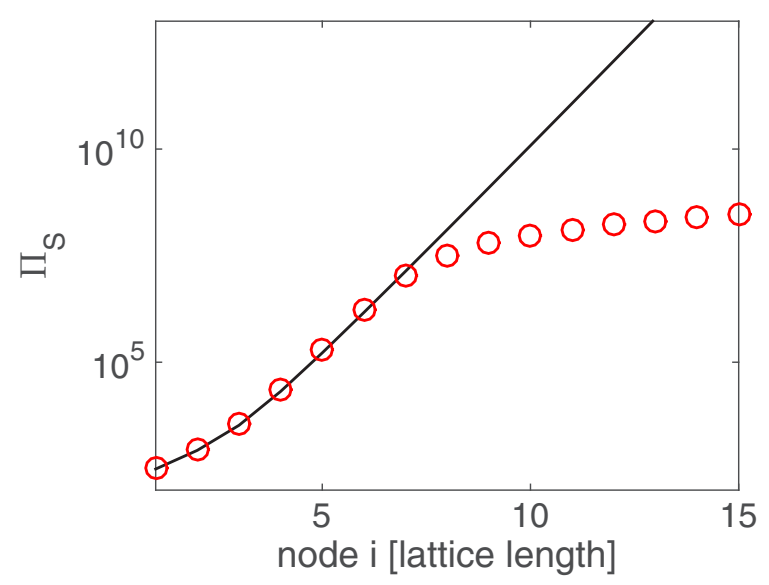

FIG. 4. $\Pi_{S}$ is plotted (solid line) vs the lattice node. The solid line refers to the analytical estimate based on linear noise approximation; see Eq. (11). Symbols refer instead to the numerical estimate based on the fully nonlinear relation (10). 
indirect measure of the lattice length. As expected, $\Pi_{S}$ grows exponentially. Symbols refer instead to a direct numerical characterization of $\Pi_{S}$, based on relation (10). Nonlinear effects induce a crossover towards a nonexponential growth for the measured entropy production rate, in complete agreement with the insight gained under a purely dynamical angle.

\section{CONCLUSION}

Stochastic quasicycles have been reported in the literature and invoked as a viable approach to the study of spontaneously generated, regular oscillations in natural systems. Stochastic oscillations are however small in amplitude. This limitation is overcome here by considering a spatially extended version of a representative reaction model that supports standard quasicycles. The asymmetry in the imposed couplings yields a nontrivial amplification mechanism that eventually results in giant cycles across the chain. To illustrate our finding we work within the context of neuroscience and adopt a hypersimplified version of the WC model. This enables us to reduce the complexity of the model to just one reaction parameter $(r)$ and one coupling constant $(D)$ : the phenomenon that we numerically demonstrate can be hence explained analytically, a task that would prove cumbersome when working with the full WC model. For the same reason we operate with a directed lattice: this is the simplest setting possible that allows us to prove our claim, while making analytical progress possible. To state it differently, the inspected model combines, within the simplest descriptive framework, the minimal ingredients that need to be accommodated for so as to yield the sought dynamics. The phenomenon is however more general and applies to other settings where the network of couplings results in a non-normal adjacency matrix [37,38], an analysis on which we shall report elsewhere.

In conclusion, we have here shown how a minimal model of neuronal population dynamics can be assembled to result in a fully tunable amplifier. The device extracts energy from the finite size bath and operates as an out of equilibrium thermal machine. This amplification process can also provide a reliable pacemaking mechanism for biological systems, beyond the specific applications discussed here. A spatially distributed detector of low intensity noisy signals can be foreseen which exploits the same, neuromorphic inspired, architecture.

\section{ACKNOWLEDGMENTS}

The authors acknowledge financial support from H2020MSCA-ITN-2015 project COSMOS 642563.

\section{APPENDIX A: STABILITY OF THE HOMOGENEOUS MEAN FIELD SOLUTION}

In the thermodynamic limit $V_{1} \rightarrow \infty$, the examined stochastic system (2) reduces to the following deterministic system:

$$
\begin{aligned}
& \frac{d}{d \tau} x_{i}=\frac{1}{\gamma_{i}}\left[f\left(s_{x_{i}}\right)-x_{i}\right], \\
& \frac{d}{d \tau} y_{i}=\frac{1}{\gamma_{i}}\left[f\left(s_{y_{i}}\right)-y_{i}\right],
\end{aligned}
$$

which, as stated in the main body of the paper, admits $x_{i}=$ $y_{i}=1 / 2 \forall i$ as a homogenous fixed point. To determine the stability of the system we carry out the linear stability analysis ${ }^{2}$ and obtain

$$
\boldsymbol{J}=\left(\begin{array}{ccccc}
\boldsymbol{E}_{1} & 0 & 0 & 0 & 0 \\
\boldsymbol{S}_{2} & \boldsymbol{E}_{2} & 0 & 0 & 0 \\
0 & \boldsymbol{S}_{3} & \boldsymbol{E}_{3} & 0 & 0 \\
0 & 0 & \ddots & \ddots & 0 \\
0 & 0 & 0 & \boldsymbol{S}_{\Omega} & \boldsymbol{E}_{\Omega}
\end{array}\right),
$$

where

$$
\begin{gathered}
\boldsymbol{E}_{1}=\left(\begin{array}{cc}
-1 & -\frac{r}{4} \\
\frac{r}{4} & -1
\end{array}\right), \\
\boldsymbol{E}_{i}=\left(\begin{array}{cc}
-\frac{1+D / 4}{\gamma_{i}} & -\frac{r-D}{4 \gamma_{i}} \\
\frac{r-D}{4 \gamma_{i}} & -\frac{1-D / 4}{\gamma_{i}}
\end{array}\right), \\
\boldsymbol{S}_{i}=\frac{D}{4 \sqrt{\gamma_{i} \gamma_{i-1}}}\left(\begin{array}{cc}
1 & -1 \\
1 & -1
\end{array}\right) .
\end{gathered}
$$

The characteristic polynomial of $\boldsymbol{J}$ is written $0=\operatorname{det}(J-$ $\lambda I)=\operatorname{det}\left(E_{1}-\lambda I\right) \prod_{i=2}^{\Omega} \operatorname{det}\left(E_{i}-\lambda I\right)$. The first term in the preceding expression gives a quadratic equation for $\lambda,(\lambda+$ $1)^{2}+\frac{r^{2}}{16}=0$. This latter yields $\lambda_{1,2}=-1 \pm i \frac{r}{4} \equiv-1 \pm i \omega_{0}$. The remaining eigenvalues are obtained by solving the following $\Omega$ equations:

$$
\left(\frac{1+D / 4}{\gamma_{i}}+\lambda\right)\left(\frac{1-D / 4}{\gamma_{i}}+\lambda\right)+\frac{(r-D)^{2}}{16 \gamma_{i}^{2}}=0,
$$

allowing one to immediately obtain

$$
\left(\lambda_{i}\right)_{3,4}=\frac{1}{\gamma_{i}}\left[-1 \pm \sqrt{-\frac{r}{8}\left(\frac{r}{2}-D\right)}\right]
$$

Notice that, for $V_{i}=V_{1} \forall i$ (or, equivalently, $\gamma_{i}=1, \forall i$ ), $\left(\lambda_{i}\right)_{3,4} \equiv \lambda_{3,4}$, as stated in the main body of the paper. By additionally requiring $D<r / 2, \lambda_{3,4}=-1 \pm i \omega_{1}$, with $\omega_{1}=$ $\sqrt{\frac{r}{8}\left(\frac{r}{2}-D\right)}$.

\section{Computing the moments of the Gaussian multivariate distribution $\Pi$}

We shall here derive the dynamical equations that control the evolution of the moments of the distribution $\Pi$. Focus on the first moment, by multiplying Eq. (6) by $\zeta_{k}$ and integrating over $\zeta$. The left hand side of the equation yields

$$
\int d \zeta \zeta_{k} \frac{\partial}{\partial \tau} \Pi=\int d \zeta \frac{\partial}{\partial \tau} \Pi \zeta_{k}=\frac{d}{d \tau} \int d \zeta \zeta_{k} \Pi=\frac{d}{d \tau}\left\langle\zeta_{k}\right\rangle
$$

The right hand side can be split into two parts. Under mild assumptions for $\Pi$, the drift term returns:

$$
-\sum_{i=1}^{2 \Omega} \int d \zeta \zeta_{k} \frac{\partial}{\partial \zeta_{i}}\left[(J \zeta)_{i} \Pi\right]
$$

\footnotetext{
${ }^{2}$ To this end, and so as to make contact with the analysis performed under the linear noise approximation, we have scaled the perturbation imposed on node $i$ by $1 / \sqrt{\gamma_{i}}$.
} 
The contribution $i=k$ amounts to

$$
\begin{aligned}
\int d \zeta \zeta_{k} \frac{\partial}{\partial \zeta_{k}}\left[(J \zeta)_{k} \Pi\right] & =\int \prod_{j \neq k} d \zeta_{j} \int d \zeta_{k} \zeta_{k} \frac{\partial}{\partial \zeta_{k}}\left[(J \zeta)_{k} \Pi\right] \\
& =-\int \prod_{j \neq k} d \zeta_{j} \int d \zeta_{k}\left[(J \zeta)_{k} \Pi\right] \\
& =-\int d \zeta\left[(J \zeta)_{k} \Pi\right]=-\left\langle(J \zeta)_{k}\right\rangle,
\end{aligned}
$$

while the terms with $i \neq k$ give no contributions. In fact,

$$
\int \prod_{j \neq k, i} d \zeta_{j} \int d \zeta_{k} \zeta_{k} \int d \zeta_{i} \frac{\partial}{\partial \zeta_{i}}\left[(J \zeta)_{i} \Pi\right]=0 .
$$

It is then straightforward to conclude that the diffusion terms return no contributions, because $\Pi$ decays fast enough at the boundaries. Summing up, we therefore obtain the linear equations:

$$
\frac{d}{d \tau}\left\langle\zeta_{k}\right\rangle=\left\langle(J \zeta)_{k}\right\rangle=\sum_{j=1}^{2 \Omega} J_{k j}\left\langle\zeta_{j}\right\rangle
$$

The unique stationary (stable) solution is therefore $\left\langle\zeta_{k}\right\rangle=$ $0 \quad \forall k$.

An identical procedure can be followed to evaluate the second moments of the distribution, namely $\left\langle\zeta_{l} \zeta_{m}\right\rangle$. To this end we multiply Eq. (6) by $\zeta_{l} \zeta_{m}$ and integrate over $\zeta$. In analogy with the above, the left hand side of the equation returns:

$$
\int d \zeta \zeta_{l} \zeta_{m} \frac{\partial}{\partial \tau} \Pi=\frac{d}{d \tau}\left\langle\zeta_{l} \zeta_{m}\right\rangle
$$

When it comes to the drift term, we shall focus first on the diagonal, $l=m$, contributions:

$$
-\sum_{i=1}^{2 \Omega} \int d \zeta \zeta_{l}^{2} \frac{\partial}{\partial \zeta_{i}}\left[(J \zeta)_{i} \Pi\right]
$$

For $i=l$, we get

$$
\begin{aligned}
\int d \zeta \zeta_{l}^{2} \frac{\partial}{\partial \zeta_{l}}\left[(J \zeta)_{l} \Pi\right] & =\int \prod_{j \neq l} d \zeta_{j} \int d \zeta_{l} \zeta_{l}^{2} \frac{\partial}{\partial \zeta_{l}}\left[(J \zeta)_{l} \Pi\right] \\
& =-2 \int \prod_{j \neq l} d \zeta_{j} \int d \zeta_{l} \zeta_{l}(J \zeta)_{l} \Pi \\
& =-2\left\langle\zeta_{l}(J \zeta)_{l}\right\rangle,
\end{aligned}
$$

while for $i \neq l$ one finds

$$
\begin{aligned}
\int d \zeta \zeta_{l}^{2} \frac{\partial}{\partial \zeta_{i}}\left[(J \zeta)_{i} \Pi\right]= & \int \prod_{j \neq l, i} d \zeta_{j} \int d \zeta_{l} \zeta_{l}^{2} \\
& \times \int d \zeta_{i} \frac{\partial}{\partial \zeta_{i}}\left[(J \zeta)_{i} \Pi\right]=0
\end{aligned}
$$

Consider now the contribution of the drift to the off diagonal elements $(l \neq m)$, namely,

$$
-\sum_{i=1}^{2 \Omega} \int d \zeta \zeta_{l} \zeta_{m} \frac{\partial}{\partial \zeta_{i}}\left[(J \zeta)_{i} \Pi\right]
$$

For $i=l$, one gets

$$
\begin{aligned}
& \int \prod_{j \neq l, m} d \zeta_{j} \int d \zeta_{m} \zeta_{m} \int d \zeta_{l} \zeta_{l} \frac{\partial}{\partial \zeta_{l}}\left[(J \zeta)_{l} \Pi\right] \\
& =-\int \prod_{j \neq l, m} d \zeta_{j} \int d \zeta_{m} \zeta_{m} \int d \zeta_{l}\left[(J \zeta)_{l} \Pi\right] \\
& =-\left\langle\zeta_{m}(J \zeta)_{l}\right\rangle .
\end{aligned}
$$

The other case of interest, $i=m$, is easy to treat, as it amounts to swapping $l$ and $m$. Finally, for $i \neq l, m$ the drift term returns a null contribution:

$$
\int \prod_{j \neq m, l, i} d \zeta_{j} \int d \zeta_{m} \zeta_{m} \int d \zeta_{l} \zeta_{l} \int d \zeta_{i} \frac{\partial}{\partial \zeta_{i}}\left[(J \zeta)_{i} \Pi\right]=0
$$

Let us now turn to considering the contribution of the diffusion terms in the Fokker-Planck equation. Since $\mathcal{B}$ is diagonal, a nontrivial contribution is solely found for $l=m$ :

$$
\frac{1}{2} \sum_{i=1}^{2 \Omega} \int d \zeta \zeta_{l}^{2} \frac{\partial^{2}}{\partial \zeta_{i}^{2}} \mathcal{B}_{i i} \Pi
$$

For $i=l$, we have

$$
\begin{aligned}
& \frac{1}{2} \int \prod_{j \neq l} d \zeta_{j} \int d \zeta_{l} \zeta_{l}^{2} \frac{\partial^{2}}{\partial \zeta_{l}^{2}} \mathcal{B}_{l l} \Pi \\
& \quad=-2 \frac{1}{2} \int \prod_{j \neq l} d \zeta_{j} \int d \zeta_{l} \zeta_{l} \frac{\partial}{\partial \zeta_{l}} \mathcal{B}_{l l} \Pi \\
& \quad=\int \prod_{j \neq l} d \zeta_{j} \int d \zeta_{l} \mathcal{B}_{l l} \Pi=\mathcal{B}_{l l} \int d \zeta \Pi=\mathcal{B}_{l l}
\end{aligned}
$$

where use has been made of the condition of normalization for the distribution $\Pi$. The case $i \neq l$ yields no contribution as

$$
\frac{1}{2} \int \prod_{j \neq l, i} d \zeta_{j} \int d \zeta_{l} \zeta_{l}^{2} \int d \zeta_{i} \frac{\partial^{2}}{\partial \zeta_{i}^{2}} \mathcal{B}_{i i} \Pi=0 .
$$

Collecting all terms together we end up with the equations for the second moments reported in the main body of the paper; see Eqs. (8).

\section{Analytical estimate for the leftmost boundary of the amplification domain}

Computing the moments of the multivariate Gaussian that characterize the stationary distribution of fluctuations under the linear noise approximation implies solving a $2 \Omega \times 2 \Omega$ problem. To gain analytical insight into the problem (with reference to the setting $\gamma_{i}=1$ ), one can operate a drastic simplification by solely accounting for nearest neighbors correlations. In doing so, one obtains a $7 \times 7$ linear system, which we do not write here explicitly because it involves lengthy expressions. Due to the structure of the problem, the $7 \times 7$ system rigorously reduces to an effective map, from a given node to the next one, for the reference quantities $\boldsymbol{w}_{i}=\left(\left\langle\xi_{i}^{2}\right\rangle,\left\langle\eta_{i}^{2}\right\rangle,\left\langle\xi_{i} \eta_{i}\right\rangle\right)$. More concretely, one can recast the problem in the form

$$
\boldsymbol{w}_{i+1}=\boldsymbol{A} \boldsymbol{w}_{i}+\boldsymbol{r}
$$


where $\boldsymbol{A}$ (not given here explicitly) is nondiagonalizable, it has rank 2 , and eigenvalues $0, \lambda$, with

$$
\lambda=\frac{-2 D^{3} r^{3}+D^{2} r^{4}+80 D^{2} r^{2}}{128 D^{2} r^{2}-128 D r^{3}-2048 D r+32 r^{4}+1024 r^{2}+8192} .
$$

To solve the problem one can reduce $\boldsymbol{A}$ to a Jordan normal form $\mathcal{A}$. It can be in fact shown that a matrix $\boldsymbol{P}$ exists such that $\mathcal{A}=\boldsymbol{P}^{-1} \boldsymbol{A} \boldsymbol{P}$.

By operating the change of variables $\boldsymbol{q}_{i}=\boldsymbol{P}^{-1} \boldsymbol{w}_{i}$ and defining $\boldsymbol{R}=\boldsymbol{P}^{-1} \boldsymbol{r}$ one gets

$$
\boldsymbol{q}_{i+1}=\mathcal{A} \boldsymbol{q}_{i}+\boldsymbol{R}
$$

that can be shown to yield

$$
\left\{\begin{array}{l}
q_{i+1}^{(1)}=q_{i}^{(2)}+R^{(1)}, \\
q_{i+1}^{(2)}=R^{(2)}, \\
q_{i+1}^{(3)}=\lambda q_{i}^{(3)}+R^{(3)},
\end{array}\right.
$$

where $\boldsymbol{q}_{i} \equiv\left(q_{i}^{(1)}, q_{i}^{(2)}, q_{i}^{(3)}\right)$ and $\boldsymbol{R} \equiv\left(R^{(1)}, R^{(2)}, R^{(3)}\right)$. Solving the above system and going back to the original variables, one eventually gets

$$
\begin{aligned}
\left\langle\xi_{i}^{2}\right\rangle= & P_{13}\left(q_{3}(0)+\frac{R^{(3)}}{\lambda-1}\right) \lambda^{i} \\
& +P_{11}\left(R^{(1)}+R^{(2)}\right)+P_{12} R^{(2)}-P_{13} \frac{R^{(3)}}{\lambda-1} \\
\left\langle\eta_{i}^{2}\right\rangle= & P_{23}\left(q_{3}(0)+\frac{R^{(3)}}{\lambda-1}\right) \lambda^{i} \\
& +P_{21}\left(R^{(1)}+R^{(2)}\right)+P_{22} R^{(2)}-P_{23} \frac{R^{(3)}}{\lambda-1} \\
\left\langle\eta_{n} \xi_{i}\right\rangle= & P_{33}\left(q_{3}(0)+\frac{R^{(3)}}{\lambda-1}\right) \lambda^{i} \\
& +P_{31}\left(R^{(1)}+R^{(2)}\right)+P_{32} R^{(2)}-P_{33} \frac{R^{(3)}}{\lambda-1} .
\end{aligned}
$$

The amplification is hence lost if $|\lambda| \leqslant 1$. The leftmost solid (white) line in Fig. 3 in the main body of the paper, panel (b), corresponds to the limiting condition $\lambda=1$. The boundary of the domain where the amplification takes place is adequately reproduced, an observation that supports a posteriori the validity of the approximations involved in the analysis.

\section{Amplifying the harmonics of $\omega_{0}$}

To amplify the harmonics of $\omega_{0}$ for any given $D$, within the domain deputed to the amplification, we can modulate the volumes of the nodes, following the strategy discussed below. Label $V_{1}$ the volume of the first node. Recall that $\omega_{1}=$ $\sqrt{\frac{r}{8}\left(\frac{r}{2}-D\right)}$ identifies the frequency that gets amplified when the volumes are forced to be identical, or, equivalently, when $\gamma_{i}=1 \forall i$. To instigate the emergence of a second peak in $\omega_{0} / 2$, on the second node of the lattice, one needs to impose the condition $\frac{\omega_{1}}{\gamma_{2}} \equiv \frac{\omega_{0}}{2}$ which readily translates in $V_{2}=2 V_{1} \frac{\omega_{1}}{\omega_{0}}$. To enforce the amplification of a train of successive harmonics one can expand on the above recipe and eventually obtain the following condition for the relative modulation of the volumes:

$$
V_{i}=2^{i-1} \frac{\omega_{1}}{\omega_{0}} V_{1}, \quad i \geqslant 2 .
$$

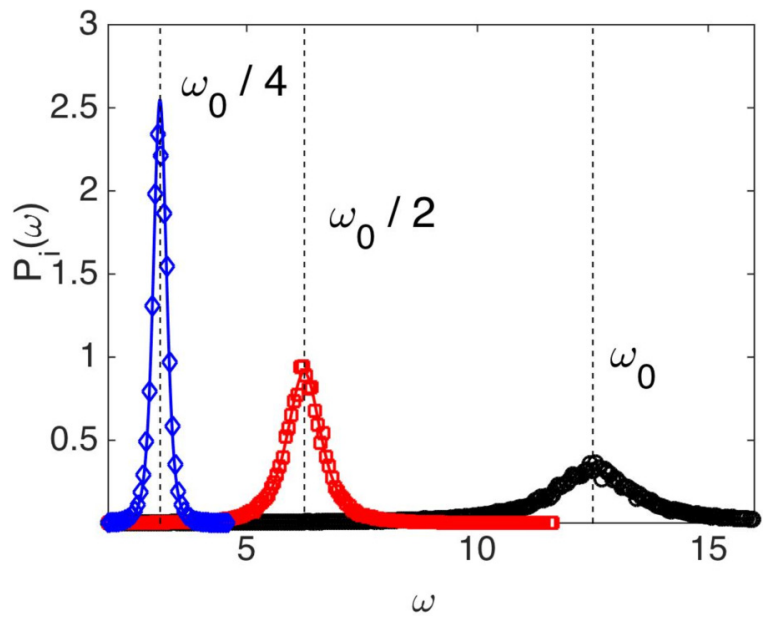

FIG. 5. Amplifying the harmonics of $\omega_{0}$, following the scheme that yields to Eq. (A2). The power spectra of fluctuations on different nodes (see text) are displayed. Symbols refer to direct simulations and the solid lines to the theory prediction.

In practice, to allow for the amplification to produce significant intensities of the signal at each frequency, one could keep the volumes constant over a few consecutive nodes, before increasing the size of the volumes of the successive set of nodes, as prescribed by formula (A2). In Fig. 5 we assumed a sequence of nodes with volumes $\left(V_{1}, V_{2}, V_{2}, V_{3}, V_{3}, V_{3}\right)$. The power spectra depicted in Fig. 5 refer to the first, third, and sixth nodes of the chain, respectively.

\section{Amplifying on a frequency comb}

We shall here demonstrate that the amplification can take place on a frequency comb. We shall in particular amplify a set of frequencies $\omega_{k}=\omega_{0}-k \Delta \omega$ with $k=0,1,2, \ldots ; \Delta \omega$ is positive and represents the relative distance between two consecutive frequency peaks. Reasoning as in the preceding

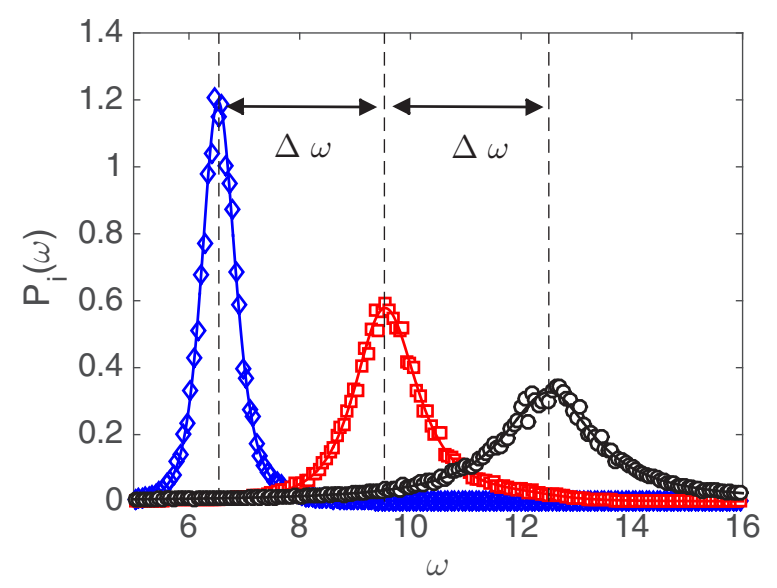

FIG. 6. Amplifying a frequency comb. Here, $\omega_{k}=\omega_{0}-k \Delta \omega$ with $k=0,1,2, \ldots$. The positive quantity $\Delta \omega$ denotes the separation between two consecutive frequencies. The size of the volumes of the nodes are set as prescribed by Eqs. (A3) and (A4). The power spectra of fluctuations on different nodes (see text) are displayed. Symbols refer to direct simulations and the solid lines to the theory prediction. 


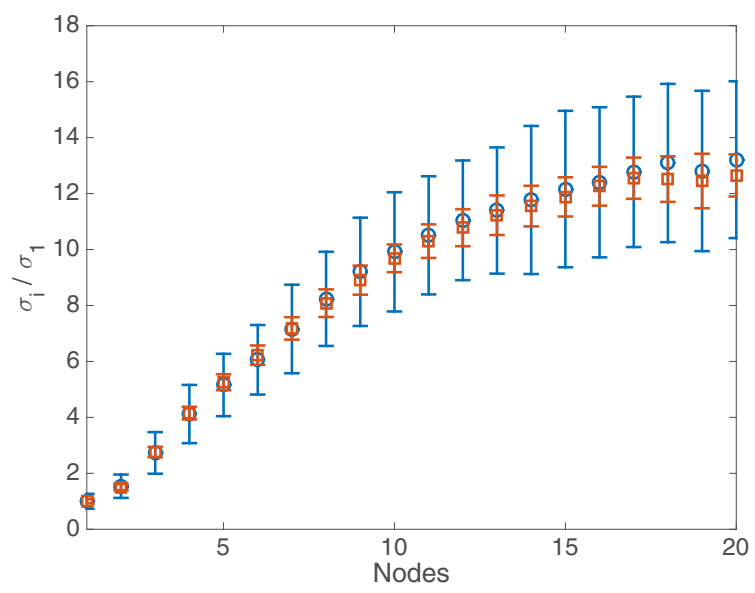

FIG. 7. $\sigma_{i} / \sigma_{1}$ vs a progressive index that identifies the nodes location. Circles refer to Gillespie based simulations and squares to a direct integration of the nonlinear Langevin equations [Eqs. (2) in the main paper]. The error bars are obtained by averaging over different realizations of the stochastic dynamics. Here, $D=10, r=50$, and $V=20000$.

section, we want to assign the volume of the second node so as to meet the condition $\frac{\omega_{1}}{\gamma_{2}}-\omega_{0} \equiv-\Delta \omega$ which translates into

$$
V_{2}=\hat{V} \frac{1}{\omega_{0} / \Delta \omega-1},
$$

where $\hat{V}=\frac{\omega_{1}}{\Delta \omega} V_{1}$. Based on the same reasoning, we get for the other nodes the following recursive relation:

$$
V_{i}=\frac{V_{i-1}}{1-\frac{V_{i-1}}{\hat{V}}} .
$$

As discussed in the preceding section, one can keep the volumes unchanged over a few consecutive nodes, before modulating their size as prescribed by formulas (A3) and (A4), so as to enhance the amplification power of the device. In Fig. 6 we created a chain that implements the sequence of volumes $\left(V_{1}, V_{2}, V_{2}, V_{3}, V_{3}, V_{3}\right)$. The power spectra displayed in Fig. 6 refer to the first, third, and sixth nodes, respectively.

\section{On the validity of the Kramers-Moyal approximation: A numerical test}

We here aim at testing the adequacy of the nonlinear Langevin equations, subject to multiplicative noise, as derived

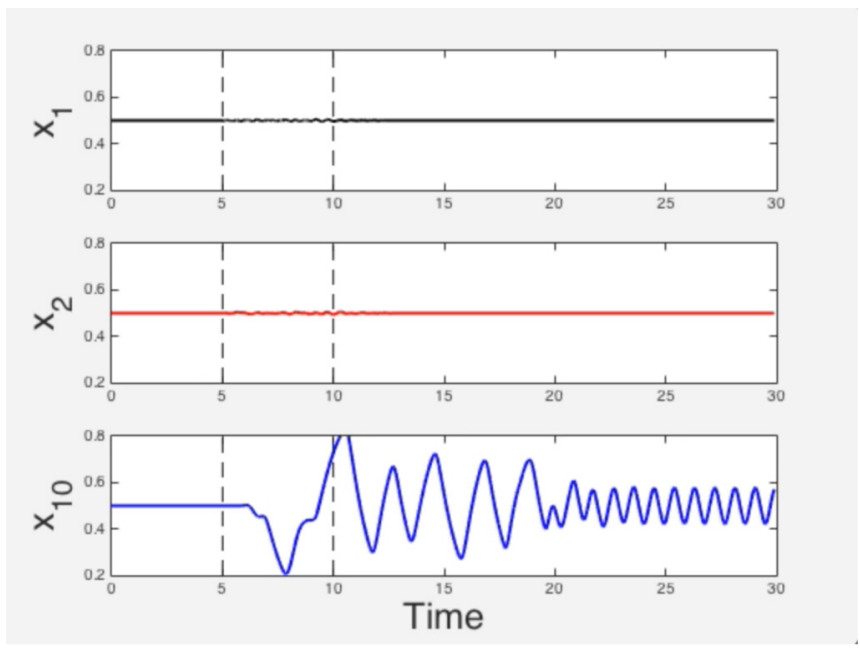

FIG. 8. Final snapshot of the dynamics as obtained in the annexed Movie 2 is displayed.

within the Kramers-Moyal picture. To this end we numerically evaluate $\sigma_{i} / \sigma_{1}$, as defined in the main body of the paper, by using (i) the Gillespie algorithm (which returns an exact description of the underlying master equation) and (ii) the nonlinear equations (see main paper). The analysis is carried out for a sufficiently small volume amount, so that Gillespie based simulations are relatively unexpensive. The comparison as drawn in Fig. 7 certifies the accuracy of the Langevin representation of the dynamics.

\section{APPENDIX B: LEGENDS OF SM MOVIES}

Movie 1: amplif 8 node1. Stochastic trajectories are shown on different nodes of the chain (respectively on nodes $1,2,8$, from upper to lower panel). The amplification mechanism as discussed in the main body of the paper is clearly displayed. Parameters are set as in Fig. 2 of the main text.

Movie 2: amplif Detection. The system is now operated in its deterministic limit. A noisy, almost undetectable, signal is injected in the first node of the chain and maintained over a finite time window (as delimited by the two vertical dashed lines). The perturbation gets magnified along the chain and materializes in a quasiregular signal in, e.g., node 10 of the collection (see lower panel). The induced oscillations display a characteristic frequency that can be tuned by acting on the coupling constant $D$. In Fig. 8 the final snapshot of the dynamics is depicted.
[1] B. Alberts, A. Johnson, J. Lewis, M. Raff, K. Roberts, and P. Walter, Molecular Biology of the Cell (Garland Science, New York, 2002).

[2] J. Macia, F. Posas, and R. V. Solé, Trends Biotechnol. 30, 342 (2012).

[3] E. R. Kandel, J. H. Schwartz, and T. M. Jessell, Principles of Neural Science, 4th ed. (McGraw-Hill, New York, 2000).

[4] P. C. Bressloff, Phys. Rev. E 82, 051903 (2010).
[5] T. C. Butler, M. Benayoun, E. Wallace, W. van Drongelen, N. Goldenfeld, and J. D. Cowan, PNAS 109, 606 (2012).

[6] A. Hutt, Front. Comput. Neurosci. 7, 2 (2013).

[7] M. Hashemi, A. Hutt, and J. Sleigh, J. Comput. Neurosci. 39, 155 (2015).

[8] N. G. van Kampen, Stochastic Processes in Physics and Chemistry (Elsevier, Amsterdam, 2007). 
[9] A. J. McKane and T. J. Newman, Phys. Rev. Lett. 94, 218102 (2005).

[10] T. Dauxois, F. Di Patti, D. Fanelli, and A. J. McKane, Phys. Rev. E 79, 036112 (2009).

[11] G. Deco, V. K. Jirsa, P. A. Robinson, M. Breakspear, and K. Friston, PLoS Comput. Biol. 4, e1000092 (2008).

[12] H. R. Wilson and J. D. Cowan, Biophys. J. 12, 1 (1972).

[13] H. R. Wilson and J. D. Cowan, Kybernetik 13, 55 (1973).

[14] A. Destexhe and T. J. Sejnowski, Biol. Cybern. 101, 1 (2009).

[15] I. Goychuk and A. Goychuk, New J. Phys. 17, 045029 (2015).

[16] E. Negahbani, D. A. Steyn-Ross, M. L. Steyn-Ross, M. T. Wilson, and J. W. Sleigh, J. Math. Neurosci. 5, 9 (2015).

[17] J. D. Cowan, J. Neuman, and W. van Drongelen, J. Math. Neurosci. 6, 1 (2016).

[18] E. Wallace, M. Benayoun, W. van Drongelen, and J. D. Cowan, Plos One 6, e14804 (2011).

[19] H. Soula and C. C. Chow, Neural Comput. 19, 3262 (2007).

[20] S. El Boustani, A. Destexhe, Neural Comput. 21, 46 (2009).

[21] A. Paffi, F. Apollonio, G. d'Inzeo, and M. Liberti, Netw., Comput. Neural Syst. 24, 99 (2013).

[22] A. Hutt, A. Longtin, and L. Schimansky-Geier, Physica D 237, 755 (2008).

[23] M. S. Bartlett, J. R. Stat. Soc. Ser. A (Gen.) 120, 48 (1957).

[24] C. Zankoc, D. Fanelli, F. Ginelli, and R. Livi, Phys. Rev. E 96, 022308 (2017).
[25] C. W. Gardiner, Handbook of Stochastic Methods (SpringerVerlag, Berlin, 2004).

[26] A. J. McKane, T. Biancalani, and T. Rogers, Bull. Math. Biol. 76, 895 (2014).

[27] T. Biancalani, L. Dyson, and A. J. McKane, Phys. Rev. Lett. 112, 038101 (2014).

[28] F. Jafarpour, T. Biancalani, and N. Goldenfeld, Phys. Rev. Lett. 115, 158101 (2015).

[29] T. Biancalani and M. Assaf, Phys. Rev. Lett. 115, 208101 (2015).

[30] P. E. Kloeden and E. Platen, Numerical Solution of Stochastic Differential Equations (Springer, Berlin, 1992).

[31] See Supplemental Material at http://link.aps.org/supplemental/ 10.1103/PhysRevE.96.062313 for legends of SM movies.

[32] D. T. Gillespie, J. Phys. Chem. 81, 2340 (1977).

[33] J. Schnakenberg, Rev. Mod. Phys. 48, 571 (1976).

[34] J. L. Lebowitz and H. Spohn, J. Stat. Phys. 95, 333 (1999).

[35] T. Tomé, Braz. J. Phys. 36, 4 (2006).

[36] T. Tomé and M. J. de Oliveira, Phys. Rev. Lett. 108, 020601 (2012).

[37] L. N. Trefethen and M. Embree, Spectra and Pseudospectra: The Behavior of Nonnormal Matrices and Operators (Princeton University Press, Princeton, NJ, 2005).

[38] M. Asllani and T. Carletti, arXiv:1706.02703. 